\title{
Individually Regulated Dual-Output IPT System Based on Current-Mode Switching Cells
}

Wang, Xiaoqiang; Xu, Jianping; Leng, Minrui; Ma, Hongbo; Dragicevic, Tomislav

Published in:

IEEE Transactions on Industrial Electronics

Link to article, DOI:

10.1109/TIE.2020.3047067

Publication date:

2021

Document Version

Peer reviewed version

Link back to DTU Orbit

Citation (APA):

Wang, X., Xu, J., Leng, M., Ma, H., \& Dragicevic, T. (2021). Individually Regulated Dual-Output IPT System Based on Current-Mode Switching Cells. IEEE Transactions on Industrial Electronics, 68(12), 12930 - 12934. https://doi.org/10.1109/TIE.2020.3047067

\section{General rights}

Copyright and moral rights for the publications made accessible in the public portal are retained by the authors and/or other copyright owners and it is a condition of accessing publications that users recognise and abide by the legal requirements associated with these rights.

- Users may download and print one copy of any publication from the public portal for the purpose of private study or research.

- You may not further distribute the material or use it for any profit-making activity or commercial gain

- You may freely distribute the URL identifying the publication in the public portal 


\title{
Individually Regulated Dual-Output IPT System Based on Current-Mode Switching Cells
}

\author{
Xiaoqiang Wang, Jianping Xu, Member, IEEE, Minrui Leng, Student Member, IEEE, Hongbo Ma, Member, IEEE, and \\ Tomislav Dragicevic, Senior Member, IEEE
}

\begin{abstract}
Based on the current-mode switching cell (CMSC), an inductive power transfer (IPT) system with dual-output is proposed in this letter. In the proposed IPT system, two CMSCs are inversely connected in series at the Rx side. Two CMSCs operate in the positive half-cycle and in the negative half-cycle of the $R x$ coil current, respectively. The dual output voltages can be regulated independently. The circuit structure of the CMSC is quite simple and helps to make the Rx side more compact. The control scheme is based on $\mathbf{R x}$-side real-time regulation and thereby wireless communication links are not required. Additionally, zero voltage switching (ZVS) is ensured for all switches. In order to verify the theoretical analysis, an experimental prototype with $20 \mathrm{~V} / 3.3 \mathrm{~A}$ and $5 \mathrm{~V} / 3 \mathrm{~A}$ outputs is fabricated, and the maximum efficiency can reach $92.8 \%$.
\end{abstract}

Index Terms - Inductive power transfer (IPT), individually regulated dual-output, current-mode switching cells. ${ }^{1}$

\section{INTRODUCTION}

$T^{2}$ NDUCTIVE power transfer (IPT) technology for wirelessly charged devices has attracted enormous attention in recent years [1]. Usually, power distribution systems with multiple independently regulated voltage/current outputs are essential for many electrical or electronic devices and platforms. As an example, the supply voltage for laptops is from $16 \mathrm{~V}$ to $20 \mathrm{~V}$. However, a voltage of $5 \mathrm{~V}$ is usually required for the USB socket [2]-[4]. As a consequence, it is of great importance to develop a high-efficiency IPT system with individually regulated dual outputs.

For a wireless power receiver, a direct way to achieve dualoutput is to cascade dc-dc converters following the IPT stage [5], [6]. However, the cascaded solution has a major drawback that all power has to be processed though both the IPT stage and the down-stream dc-dc stage, which dramatically degrades the system efficiency and increases the system volume and cost.

For single-stage IPT system with multiple outputs, multi-coil structure is used by configuring the mutual inductance between each receiver $(\mathrm{Rx})$ coil and the transmitter $(\mathrm{Tx})$ coil without

Manuscript received August 31, 2020; accepted December 13, 2020. This work was supported by the National Natural Science Foundation of China under Grant 51777176, and 61733015. (Corresponding author: Jianping $X u$ and Hongbo Ma)

X. Wang, J. Xu, M. Leng and H. Ma are with the School of Electrical Engineering, Southwest Jiaotong University, Chengdu 611756, and also with the Key Laboratory of Magnetic Suspension Technology and Maglev Vehicle, Ministry of Education, Chengdu 611756, China (e-mail: xqwang0621@163.com; jpxu-swjtu@163.com; mrleng_pece@163.com; mahongbo81@gmail.com;).

Tomislav Dragicevic is with the Center of Electric Power and Energy, Technical University of Denmark, 2800 Kgs. Lyngby, Denmark (e-mail: tomdr@elektro.dtu.dk). complex control methods [7]. However, the cross-coupling between Rx coils poses a great challenge to coil design. Besides, dual-band IPT systems with different frequencies $(\mathrm{kHz}$ range and $\mathrm{MHz}$ range) have been developed to power individual loads simultaneously [8], [9]. Unfortunately, the design of the passive network is very complex due to the crosstalk interference which needs to be effectively suppressed.

Furthermore, some novel rectifier structures are put forward to obtain individually regulated multi-output. A robust selfregulated rectifier without any switching circuitry is proposed for biomedical devices [10]. In [11], a multi-phase resonancebased boosting rectifier is presented to power different loads. However, the circuit configuration at the $\mathrm{Rx}$ side is complex and the power transfer capability is limited [10], [11].

In order to make the Rx side more compact, the current-mode switching cell (CMSC) which consists of a switch, a diode and a capacitor, is proposed for the IPT system. Two CMSCs are inversely connected in series at the Rx side, where one CMSC operates in the positive half-cycle of the Rx coil current and the other CMSC operates in the negative half-cycle of the Rx coil current. The main advantages of the proposed IPT system are summarized as follows:

1) The circuit structure of the CMSC is cost-effective and simple, and helps to making the Rx side more compact.

2) The dual output voltages can be regulated independently without auxiliary decoupling circuits. As the control scheme is based on $\mathrm{Rx}$-side real-time regulation, wireless communication links are not required.

3) The proposed IPT system can achieve zero phase angle (ZPA) with unity input power factor at full load, and all switches can realize zero voltage switching (ZVS) in the whole load range.

4) It is easy to extend the dual-output configurations to the multi-output configurations for other IPT applications, such as the light-emitting diode (LED) drivers.

\section{CiRCUIT TOPOLOGY}

The circuit diagram of the proposed IPT system is shown in Fig. 1(a). The self-inductances of Tx, Rx are $L_{\mathrm{p}}, L_{\mathrm{s}}$, and the mutual inductance is $M . k$ is the coupling coefficient of the magnetic coupler, which is defined as $k=M /\left(L_{\mathrm{p}} L_{\mathrm{s}}\right)^{1 / 2} \cdot C_{\mathrm{p}}$ and $C_{\mathrm{s}}$ are the compensation capacitors. $v_{\mathrm{ab}}$ is the equivalent output ac voltage of the primary inverter and $v_{\mathrm{s}}$ is the equivalent input ac voltage of the CMSC. The CMSC $i$ consists of a switch $\mathrm{S}_{i}$, a diode $\mathrm{D}_{i}$ and a capacitor $C_{i}$, where $i=a, b . R_{\mathrm{a}}$ and $R_{\mathrm{b}}$ are the load resistances. Fundamental harmonic approximation (FHA) method is adopted to simplify the analysis [12].

Fig. 1(b) shows the equivalent ac circuit of the proposed IPT system. The CMSC $i$ together with the load resistance $R_{i}$ can be 
represented by an equivalent ac impedance $Z_{i}$. In the following analysis, $I_{\mathrm{p}}$ represents the root-mean-square $(\mathrm{rms})$ value of $i_{\mathrm{p}}$ and other variables are denoted in a similar way.

In order to fully tune the IPT system, following equations should be satisfied [1]

$$
1 / \omega=\sqrt{L_{\mathrm{p}} C_{\mathrm{p}}}=\sqrt{L_{\mathrm{s}} C_{\mathrm{s}}}
$$

where $\omega$ is the switching angle frequency.

By using Kirchhoff voltage law (KVL), from Fig. 1, the Tx and Rx coil currents can be derived as

$$
I_{\mathrm{p}}=\frac{2 \sqrt{2} V_{\mathrm{in}}}{\pi \omega^{2} M^{2}}\left(\left|Z_{\mathrm{a}}+Z_{\mathrm{b}}\right|\right), I_{\mathrm{s}}=\frac{2 \sqrt{2} V_{\mathrm{in}}}{\pi \omega M}
$$

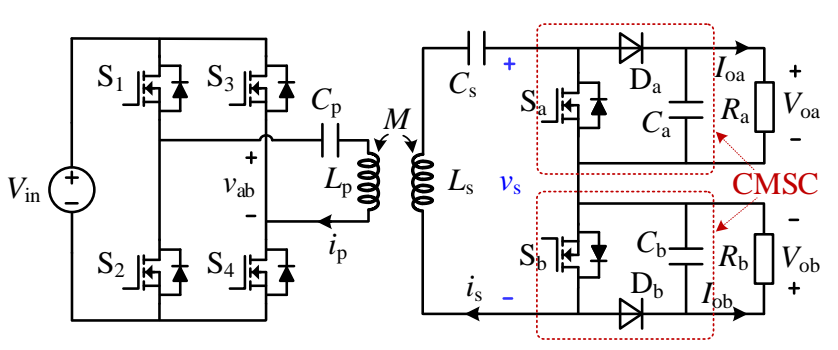

(a)

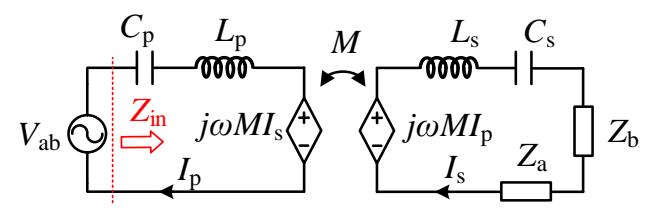

(b)

Fig. 1. Circuits of the proposed IPT system. (a) The main circuit topology, (b) the equivalent ac circuit.

It can be inferred from (2) that the Rx coil current $I_{\mathrm{S}}$ is independent of the load resistance. It can be kept constant as long as $M$ is constant since $\omega$ and $V_{\text {in }}$ are usually fixed. As a consequence, the input of the CMSC $i$ can be regarded as an ac current source.

Similarly, as shown in Fig. 1(b), the input impedance of the IPT system can be calculated as

$$
Z_{\text {in }}=\omega^{2} M^{2} /\left(Z_{\mathrm{a}}+Z_{\mathrm{b}}\right)
$$

From (3), it can be found that if $\left(Z_{a}+Z_{b}\right)$ is resistive, $Z_{\text {in }}$ is resistive as well, which means that the IPT system can achieve ZPA with unity power factor. Assuming that the impedance angle of $\left(Z_{\mathrm{a}}+Z_{\mathrm{b}}\right)$ is $\beta$, then, the input impedance of $Z_{\mathrm{in}}$ is $-\beta$. Practically, $-\beta$ should be slightly larger than 0 for ZVS of the primary inverter.

\section{Control Strategy}

The key operating waveforms of the proposed IPT system are shown in Fig. 2(a). $v_{\mathrm{s}, 1}$ is the fundamental component of $v_{\mathrm{s}}$. The duty cycle of both $S_{a}$ and $S_{b}$ are $50 \%$, and the effective control angle of the CMSC $i$ is defined as $\theta_{i}$. Since the CMSC $a$ and the CMSC $b$ are inversely connected in series at the receiver side, when the current $i_{\mathrm{s}}$ is positive, the CMSC $b$ is shorted. Similarly, the CMSC $a$ is shorted when the current $i_{\mathrm{s}}$ is negative, as shown in Fig. 2(b).

The proposed IPT system has six operating modes in one switching period. As an example, only the positive half-cycle is described as follows. Before $t_{0}$, the current $i_{\mathrm{s}}$ is positive and flows through the body diode of $S_{b}$. Therefore, $S_{b}$ is turned on with ZVS. In interval $t_{0} \sim t_{1}, \mathrm{~S}_{\mathrm{a}}$ is in on state, and the current $i_{\mathrm{s}}$ is freewheeling in the receiver side. $S_{a}$ is turned off with a time delay of $\theta_{\mathrm{a}}$ and the amplitude of $v_{\mathrm{s}}$ equals to $V_{\text {oa. }}$.

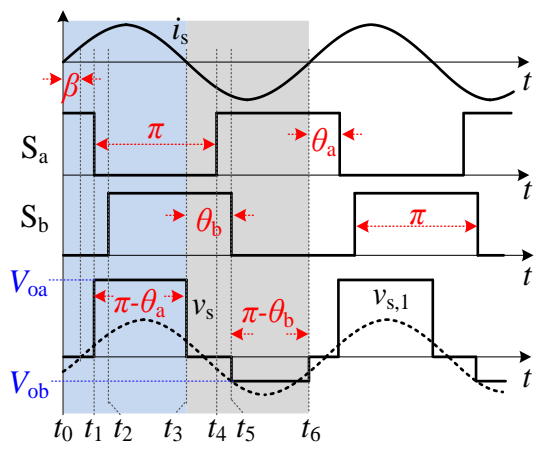

(a)

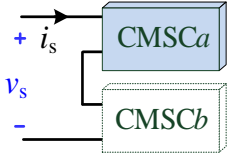

Positive half-cycle of $i_{\mathrm{s}}$

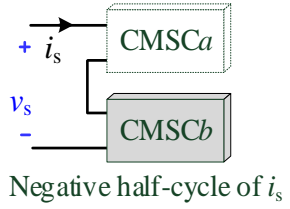

(b)
Fig. 2. Diagram of the proposed IPT system. (a) The key operating waveforms, (b) the operation mode in positive and negative half-cycle of $i_{\mathrm{s}}$.

During the time for which the current freewheels through the switch, no power is transferred to the load. Considering the current $i_{\mathrm{s}}$ is load-independent, the conduction loss of Rx coil, which plays a dominant part in the power consumption of Rx side, is almost constant. Therefore, the impact of freewheeling duration on the system efficiency is insignificant because the on-state resistance of $\mathrm{S}_{\mathrm{a}}\left(\mathrm{S}_{\mathrm{b}}\right)\left(\mathrm{BSC} 010 \mathrm{~N} 04 \mathrm{LS}, R_{\mathrm{ds}(\mathrm{on}), \max }=1 \mathrm{~m} \Omega\right)$ is quite small.

According to (2) and Fig. 2, the output currents $I_{\mathrm{oa}}$ and $I_{\mathrm{ob}}$ can be calculated as

$$
I_{\mathrm{oa}}=\frac{2 V_{\mathrm{in}}}{\pi^{2} \omega M}\left(1+\cos \theta_{\mathrm{a}}\right), I_{\mathrm{ob}}=\frac{2 V_{\mathrm{in}}}{\pi^{2} \omega M}\left(1+\cos \theta_{\mathrm{b}}\right)
$$

From (4), it can be known that the two output currents can be regulated separately. As a result, the rated voltage difference between two loads has no influence on the effectiveness of the proposed IPT system. In addition, it is noted that the variation of $\theta_{\mathrm{a}}$ and $\theta_{\mathrm{b}}$ will affect the phase angle between $v_{\mathrm{s}}$ and $i_{\mathrm{s}}$, i.e. $\beta$, as shown in Fig. 2(a). Using the Fourier decomposition, the expression of $v_{\mathrm{s}, 1}$ can be derived as

$$
v_{\mathrm{s}, 1}(t)=a_{1} \cos \omega t+b_{1} \sin \omega t
$$

where

$$
\begin{aligned}
& a_{1}=\left(V_{\mathrm{ob}} \sin \theta_{\mathrm{b}}-V_{\mathrm{oa}} \sin \theta_{\mathrm{a}}\right) / \pi \\
& b_{1}=\left[V_{\mathrm{oa}}\left(1+\cos \theta_{\mathrm{a}}\right)-V_{\mathrm{ob}}\left(1+\cos \theta_{\mathrm{b}}\right)\right] / \pi
\end{aligned}
$$

Therefore, the impedance angle of $\left(Z_{\mathrm{a}}+Z_{\mathrm{b}}\right)$ can be derived as

$$
\beta=\arctan \frac{\sin \theta_{\mathrm{b}}-\lambda \sin \theta_{\mathrm{a}}}{\lambda\left(1+\cos \theta_{\mathrm{a}}\right)-\left(1+\cos \theta_{\mathrm{b}}\right)}
$$

where $\lambda$ is the ratio of $V_{\mathrm{oa}}$ and $V_{\mathrm{ob}}$.

According to (7), the impedance angle $-\beta$ versus different $\theta_{\mathrm{a}}$ and $\theta_{\mathrm{a}}$ is shown in Fig. 3. Usually, $-\beta$ is selected as $0^{\circ} \sim 20^{\circ}$ for ZVS of the primary inverter. Although $-\beta>20^{\circ}$ is able to realize ZVS, the reactive power will be increased accordingly, which results in the reduction of the system efficiency.

From Fig. 3(a), it can be seen that the variation of $-\beta$ is less sensitive to $\theta_{\mathrm{b}}$. To realize ZVS with minimum reactive power, it is preferred to make $\theta_{\mathrm{a}}$ locate within the range of $[\pi / 12, \pi / 4]$, as shown in Fig. 3(b). Moreover, it should be emphasized that hard switching may occur when $\theta_{\mathrm{a}}$ becomes large, namely, the CMSC $a$ is under light load. In addition, it can be found from (7) that the impedance angle $\beta$ highly depends on the voltage ratio 
between two channels. Therefore, Fig. 3 is not a general result for different output voltage ratios. (a)

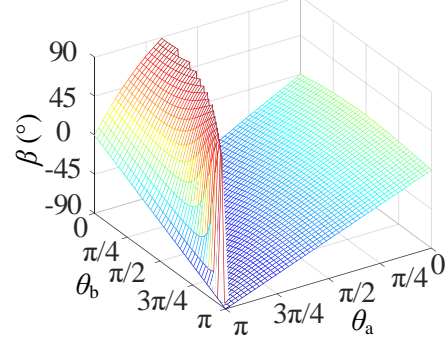

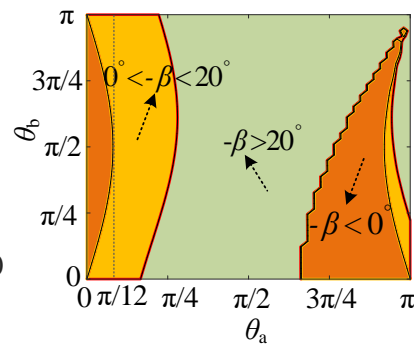

(b)
Fig. 3. The input impedance angle $\beta$ versus different $\theta_{\mathrm{a}}$ and $\theta_{\mathrm{a}}$, where $\lambda=4$. (a) The 3D surface of $\beta$, (b) the contour of $-\beta$.

The control diagram of the proposed IPT system is shown in Fig. 4. Two separate PI compensators are applied to correct the difference between $V_{\mathrm{o} i}$ and $V_{\mathrm{oi} \_ \text {ref, }}$ which generates the corresponding control angle $\theta_{i}$. It should be emphasized that the detection of $i_{\mathrm{s}}$ is required to generate a synchronization signal for the ePWM modules.

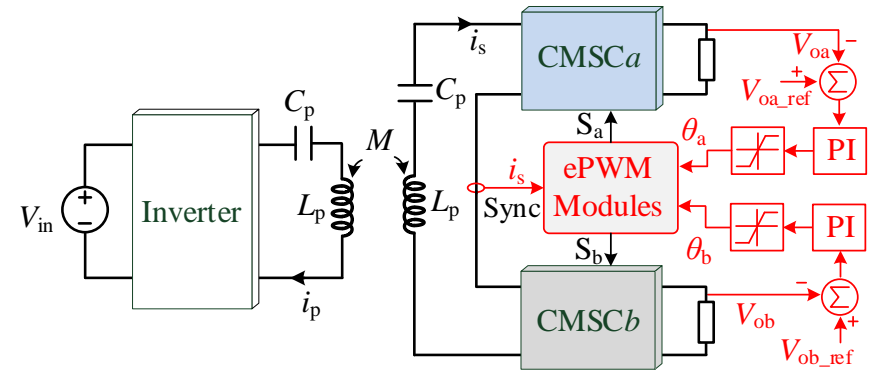

Fig. 4. The control diagram of the proposed IPT system.

\section{EXPERIMENTAL VERIFICATIONS}

To verify the proposed IPT system, an experiment prototype with 20V/3.3A and 5V/3A outputs, as shown in Fig. 5, is built. The diameter of the Tx and Rx coils are both $100 \mathrm{~mm}$ and the air gap is $30 \mathrm{~mm}$. Ten ferrite bars in each side are used to enhance the coupling. In order to obtain the required magnetic coupler with the size limitation, finite element analysis (FEA) software ANSYS is used to assist the parameter design. The flowchart of the parameters design is illustrated in Fig. 6. According to the design procedure, the electrical parameters of the IPT system are given in Table I.

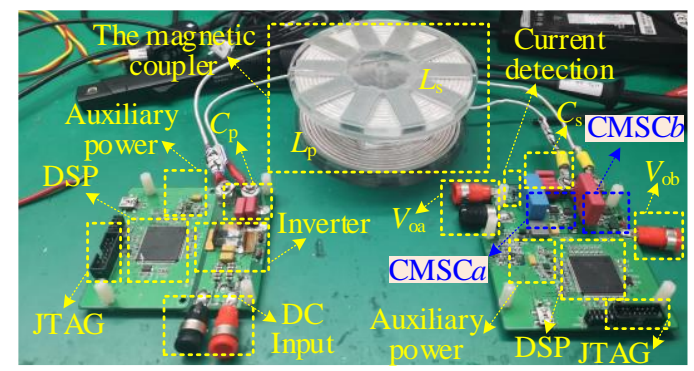

Fig. 5. The photo of the experiment prototype.

Because the switching frequency reaches up to $100 \mathrm{kHz}$, zero-crossing comparation method is commonly employed [13], [14]. The hardware synchronization circuits for the CSMCs are designed in Fig. 7. To suppress the circuit noise and time delay, bandpass filter and phase-shift circuit are adopted. Finally, the synchronization signal can be acquired accurately by the eCAP module of DSP controller.

In addition, the output voltage sampling period is $10 \mu$ s in the experiment prototype. To suppress the voltage sampling error caused by circuit noises, averaging filtering algorithm is utilized to improve the reliability of PID controller. As a result, the control period is set as $100 \mu \mathrm{s}$ (ten-times the sampling period) in the experiments.

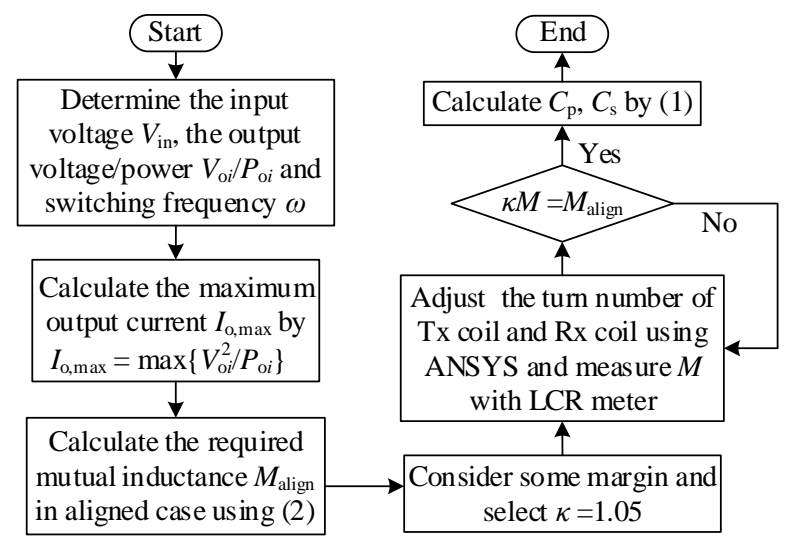

Fig. 6. The flowchart of the parameters design.

TABLE I

ELECTRICAL PARAMETERS OF THE PROPOSED IPT SYSTEM

\begin{tabular}{cccccc}
\hline \hline$V_{\text {in }}$ & $\omega$ & $L_{\mathrm{p}}$ & $C_{\mathrm{p}}$ & $L_{\mathrm{s}}$ & $C_{\mathrm{s}}$ \\
\hline $28 \mathrm{~V}$ & $2 \pi \times 100 \mathrm{kHz}$ & $17.9 \mu \mathrm{H}$ & $141.5 \mathrm{nF}$ & $17.2 \mu \mathrm{H}$ & $147.3 \mathrm{nF}$ \\
\hline$k$ & $V_{\text {oa }}$ & $V_{\text {ob }}$ & $I_{\text {oa }}$ & $I_{\text {ob }}$ & - \\
\hline $0.2-0.3$ & $20 \mathrm{~V}$ & $5 \mathrm{~V}$ & $3.3 \mathrm{~A}$ & $3 \mathrm{~A}$ & - \\
\hline \hline
\end{tabular}

Fig. 8 shows the steady-state waveforms of the proposed IPT system when the dual outputs are both under full load. It can be seen that ZVS for the primary inverter and the CMSCs can be ensured. The control angles $\theta_{\mathrm{a}}$ and $\theta_{\mathrm{b}}$ are about $36.7^{\circ}$ and $51.2^{\circ}$, respectively. Besides, ZPA of the IPT system is nearly achieved, which agrees with the analysis in Fig. 3.

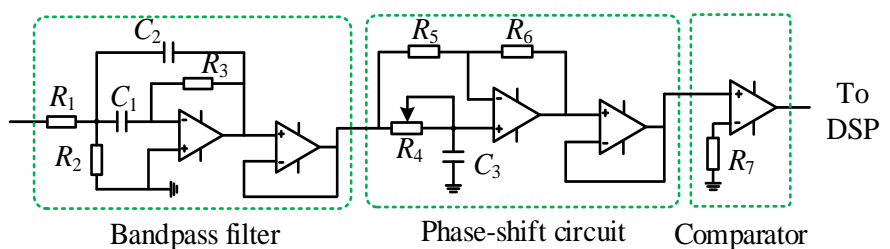

Fig. 7. The hardware synchronization circuits for the CSMCs.

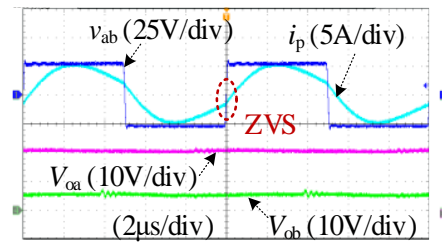

(a)

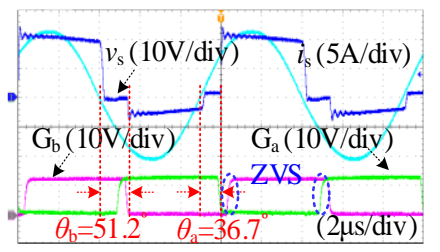

(b)
Fig. 8. Experimental waveforms of. (a) $v_{\mathrm{ab}}, i_{\mathrm{p}}, V_{\mathrm{oa}}$ and $V_{\mathrm{ob}}$, (b) $v_{\mathrm{s}}, i_{\mathrm{s}}, \mathrm{G}_{\mathrm{a}}$ and $\mathrm{G}_{\mathrm{b}}$, where $G_{a}$ and $G_{b}$ are the drive signals of $S_{a}$ and $S_{b}$, respectively.

To validate the dynamic response of the dual-output without cross regulation, load step for one output is carried out when the other output maintains constant, as shown in Fig. 9. Obviously, the dual-output will not affect each other and the decoupling 
performance of the proposed IPT system is thus verified.

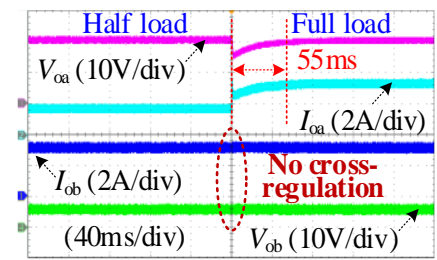

(a)

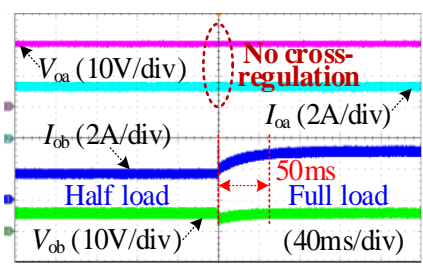

(b)
Fig. 9. Experimental waveforms. (a) The load of CMSC $a$ changes from half load to full load, (b) the load of CMSC $b$ changes from half load to full load.

Practically, coil misalignment is inevitable, which results in the variation of the coupling coefficient $k$. Fig 10 shows the waveforms of the IPT system when the coupling coefficient $k$ varies from 0.3 to 0.2 . It can be seen that $\theta_{\mathrm{a}}$ increases from $35.7^{\circ}$ to $78.5^{\circ}$ and $\theta_{\mathrm{b}}$ increases from $46.1^{\circ}$ to $82.2^{\circ}$. That is because $i_{\mathrm{s}}$ increases gradually with the decrease of $k$. Meanwhile, the input impedance angle $-\beta$ increases from $12.6^{\circ}$ to $27.7^{\circ}$, which is in accordance with the theoretical analysis.

The system efficiency is measured by the power analyzer PW6001, as shown in Fig. 11. It should be noted that the power losses of the DSPs, the driver circuits and the sampling circuits, etc. are included because the auxiliary power in Tx side and $\mathrm{Rx}$ side are converted from the DC input supply and the CMSC $a$, respectively. It can be found from Fig. 9 that the maximum efficiency is $92.8 \%$ at full load when $k=0.3$. When $k=0.2$, the increase of Rx coil current results in increase of conduction losses. Thus, the system efficiency decreases accordingly with $k=0.2$.

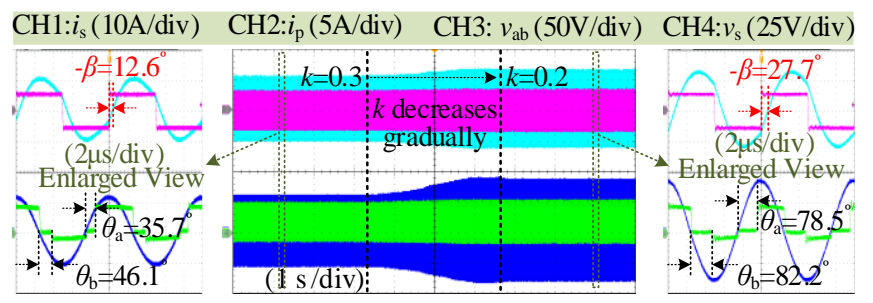

Fig. 10. Experimental waveforms when $k$ varies from 0.3 to 0.2 .

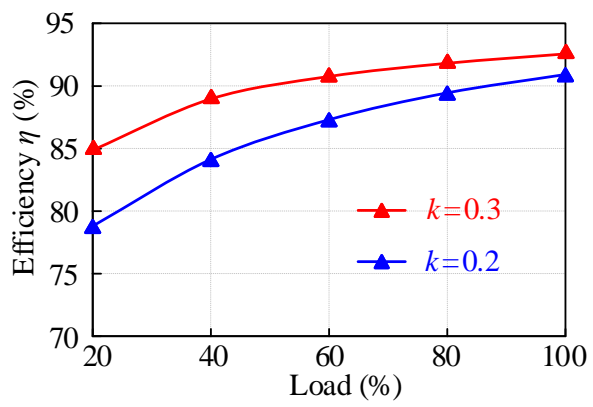

Fig. 11. The measured system efficiency under different load condition.

For more clarity on the power loss distribution of the dualoutput IPT system, the measured results with $k=0.3$ in the full load condition are depicted in Fig. 12. It can be found that the power loss of primary inverter is only $0.82 \mathrm{~W}$ because of ZVS operation and low on-state resistance. The power loss of Rx (including $L_{\mathrm{s}}$ and $C_{\mathrm{s}}$ ) is much higher than that of Tx (including $L_{\mathrm{p}}$ and $C_{\mathrm{p}}$ ) because $I_{\mathrm{s}}$ is almost twice as $I_{\mathrm{p}}$, as indicated in Fig. 8. Besides, the power losses of CMSC $a$ and $\mathrm{CMSC} b$ are $1.15 \mathrm{~W}$ and $0.85 \mathrm{~W}$, respectively. In order to further reduce the power losses of CMSC $i$, the diodes can be replaced by MOSFETs with low on-state resistance.

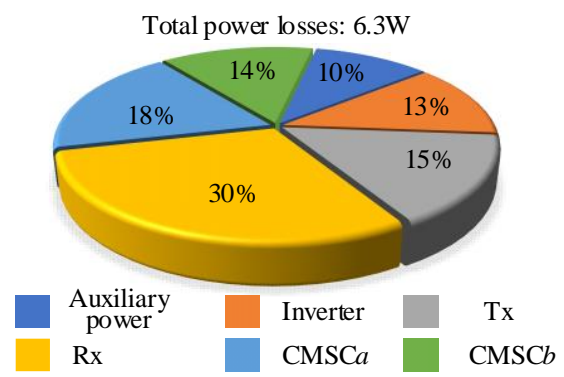

Fig. 12. The measured power loss distribution of the dual-output IPT system.

In addition, the proposed individually regulated dual-output IPT system can be extend to multiple output configurations, and the control strategy is applicable as well. As an example, for the light-emitting diode (LED) driver, Fig. 13 shows the universal $n$ output configuration with individual regulation. To make full use of the Rx coil current, it is preferred to make the number of outputs operating in the positive half-cycle be the same as that in the negative half-cycle when $n$ is an even number. When $n$ is an odd number, $(n-1) / 2$ output operate in the positive (or negative) half-cycle of Rx coil current while the others operate in the negative (or positive) half-cycle of Rx coil current.

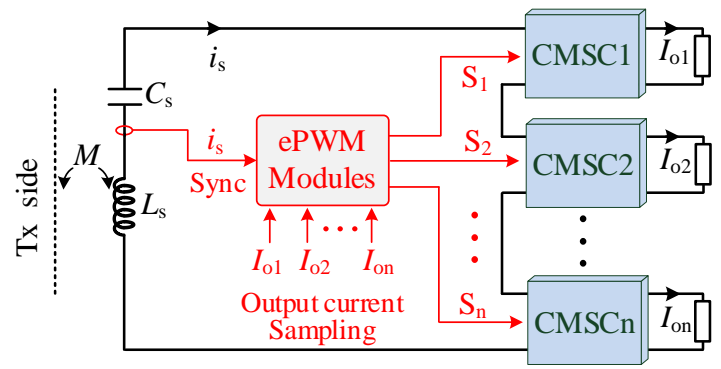

Fig. 13. The multiple output configuration with individual regulation.

\section{CONCLUSION}

In this letter, an inductive power transfer (IPT) system with dual-output is presented. Two current-mode switching cells (CMSCs) are inversely connected in series at the receiver side. As one CMSC operates in the positive half-cycle of the Rx coil current and the other CSMC operates in the negative half-cycle of the Rx coil current, the dual output voltages can be regulated independently. Additionally, the circuit structure of the CMSC is quite simple and contributes to making the $\mathrm{Rx}$ side more compact. Finally, an experimental prototype with 20V/3.3A and $5 \mathrm{~V} / 3 \mathrm{~A}$ outputs is built to verify the proposed IPT system and the experimental results show that the maximum efficiency can reach $92.8 \%$ at full load when $k=0.3$.

\section{REFERENCES}

[1] Z. Zhang, H. Pang, A. Georgiadis and C. Cecati, "Wireless power transfer-an overview," IEEE Trans. Ind. Electron., vol. 66, no. 2, pp. 1044-1058, Feb. 2019.

[2] Y. Chen, H. Wang and Y. Liu, "Improved hybrid rectifier for 1-MHz LLC-based universal AC-DC adapter," 2017 IEEE Applied Power Electronics Conference and Exposition (APEC), Tampa, FL, 2017, pp. 23-30.

[3] C. Kim, J. Baek and J. Lee, "High-efficiency single-stage LLC resonant converter for wide-input-voltage range," IEEE Trans. Power Electron., 
vol. 33, no. 9, pp. 7832-7840, Sept. 2018.

[4] Y. Cao and J. A. A. Qahouq, "Analysis and evaluation of a dual-variable closed-loop control of power converter with wireless and nonwireless power transfer," IEEE Trans. Ind. Electron., vol. 66, no. 4, pp. 2668-2679, Apr. 2019.

[5] P. Patra, J. Ghosh, and A. Patra, "Control scheme for reduced cross regulation in single-inductor multiple-output DC-DC converters," IEEE Trans. Ind. Electron., vol. 60, no. 11, pp. 5095-5104, Nov. 2013.

[6] X. Jing, P. K. T. Mok, M. C. Lee, "A wide-load-range constant-chargeauto-hopping control single-inductor-dual-output boost regulator with minimized cross-regulation", IEEE J. Solid-State Circuits, vol. 46, no. 10, pp. 2350-2362, Oct. 2011.

[7] J. J. Casanova, Z. N. Low and J. Lin, "A loosely coupled planar wireless power system for multiple receivers," IEEE Trans. Ind. Electron., vol. 56, no. 8, pp. 3060-3068, Aug. 2009.

[8] M. Liu and M. Chen, "Dual-band wireless power transfer with reactance steering network and reconfigurable receivers," IEEE Trans. Power Electron., vol. 35, no. 1, pp. 496-507, Jan. 2020.

[9] C. Zhao and D. Costinett, "GaN-Based dual-mode wireless power transfer using multifrequency programmed pulse width modulation," IEEE Trans. Ind. Electron., vol. 64, no. 11, pp. 9165-9176, Nov. 2017.

[10] N. U. Hassan, S. Hong and B. Lee, "A robust multi-output self-regulated rectifier for wirelessly-powered biomedical applications," IEEE Trans. Ind. Electron., doi: 10.1109/TIE.2020.2988226.

[11] J. Lim, B. Lee and M. Ghovanloo, "A multiphase resonance-based boosting rectifier with dual outputs for wireless power transmission," IEEE Trans. Power Electron., vol. 35, no. 3, pp. 2680-2689, Mar. 2020.

[12] X. Wang, J. Xu, H. Ma and Y. Zhang, "A reconstructed S-LCC topology with dual-type outputs for inductive power transfer systems," IEEE Trans. Power Electron., vol. 35, no. 12, pp. 12606-12611, Dec. 2020. 